\title{
A NEW APPROACH TO THE PROBLEM OF REMOVAL OF SOLUBLE GASEOUS POLLUTANTS FROM THE ATMOSPHERE AND ITS CORRESPONDENCE WITH THE EXISTING APPROACH
}

\author{
SOUMITRA CHATTOPADHYAY and A.K. MUKHERJEE \\ Science Department, Columbia College, Columbia, Missouri 65216 \\ (Received September 1, 1992)
}

\begin{abstract}
A new approach has been developed using the kinetic theory for the dissolution of gaseous pollutants in cloud droplets. The existing theory, using the diffusion approach, assumes dissolving gas to be a continuous medium. This approach may not always be suitable; especially, as the mean free paths of the pollutant gases at the existing concentrations available at the atmosphere are of the same order as the diameter of the cloud droplets. We develop an equation by considering the mass of the gas molecules striking per unit area of the droplets. Equations are also developed for the rate of dissolution of gaseous air pollutants and an attempt has been made to find out the correspondence with the classical approach and the mass transfer process.
\end{abstract}

KEY WORDS AND PHRASES. Atmospheric pollution, kinetic theory of gases, diffusion, growth and decay of a raindrop

1992 AMS SUBJECT CLASSIFICATION CODES. 76E99, 80A10, $80 \mathrm{~A} 30,00 \mathrm{~A} 69,00 \mathrm{~A} 89,82 \mathrm{~A} 40$, $86 \mathrm{~A} 10,86 \mathrm{~A} 35$.

\section{INTRODUCTION.}

The kinetics of formation and evaporation of cloud droplets has been a matter of interest to scientists for a long time. Maxwell [1] (1890) discussed the kinetics of these two processes in his famous paper where a continuity in the temperature field at the surface of the droplets was assumed. The system was considered to be at a constant temperature. The equation by Maxwell was later modified by Mason [2], Neiberger [3] and Jiusto et al [4] in the late fifties and the sixties, for the growth and evaporation of cloud droplets.

The subject of pollution of cloud droplets is interesting in its own right as it may give rise to severe atmospheric pollution including the causing of acid rain. A cloud droplet may become polluted due to a number of reasons. Pollutants entering a droplet are mainly in two forms: (i) gaseous pollutants and (ii) particulate matters or aerosols. The main difference between these two types lies in the fact that the dissolution of gaseous pollutants in rain is a reversible process whereas that of the particulates is not. The current theory proposed by Hales [5] for gas scavenging is based on the assumption that in the process of the dissolution of gases by rain, there is a gradient of concentration of gases in the ambient air upto a certain distance from the droplet dissolving the gas. The theory also assumes that the dissolution occurs by a diffusion process.

In 1968, Engelmann [6] summarized the efforts of all previous significant advancements in the 
scavenging theory till that time. Later, Fuquay [7] noted that most of the authors interested in the subject of gas scavenging by a raindrop had speculated that trace pollutant gases dissolved in the raindrops were totally soluble, and derived equations assuming irreversible wash-out process. But these considerations are not necessarily true. Junge [8], Postma [9], and Hales et al [10] have concluded that the total solubility of pollutants in a raindrop is an impractical proposition.

The basic assumption in the mechanism of the diffusion approach is that the pollutant gases form a continuous medium. Again, this assumption, for all practical purposes, is valid only for high partial pressures of gases. In case of cloud droplets, it can be easily realized that the gases entering the solution may have mean free paths of the order of the diameter of the cloud droplets. Therefore, the gaseous medium concerned is not always continuous. Hence, the validity of the diffusion approach becomes questionable at the pressure and temperature of the atmosphere.

\section{THE NEW APPROACH.}

In this paper, we propose a new approach to deal with the situation by applying the kinetic theory. The main emphasis of this work is on the rain-out process. But this idea can be extended to be applied to wash-out also after due allowance is given to the aerodynamics of a falling droplet and the effects of ventilation. We consider the gas medium to be a discontinuous one containing molecules and empty spaces. In course of their translatory motion, these molecules and empty spaces hit the surface of the cloud droplets, in accordance with the kinetic theory. As a result, some of the molecules will be captured by the droplets while the others will simply rebound. As saturation is approached, the number of molecules captured will decrease as the concentration of the dissolved gas increases. Also, the number of droplets will decrease as time goes on. In this work, we will relate the rate of change of concentration of a droplet (i) with the size (radius) of the droplet (i.e. the growth and/or evaporation of the droplet) and (ii) with time.

Hales [5] proposed that this phenomenon may be mathematically approached by using the analysis of mass-transfer. The kinetic theory approach presented here is a more general approach. We will show that on a large time scale involving a large number of molecules, our model yields the same results as the other approach as the concentration of the pollutant gases becomes high.

\section{GAS SCAVENGING.}

Fukuta and Walter [11] first described the growth of a cloud droplet using the kinetic theory approach. Later Mukherjee [12] proposed that the gas scavenging can be approached from kinetic theory also, but no comparison was made between the new approach and the diffusion approach. Before Mukherjee's proposition, the problem was approached using Hales' [5] prescription that assumes the lowering of the wash-out rate as precipitation becomes saturated with respect to the pollutant gas. It was also assumed by Hales that a falling raindrop may become supersaturated with respect to the ambient temperature if the circumstances are favorable.

In all our following discussions, we will assume a droplet to be of spherical shape, in accordance with one of the basic assumptions of the kinetic theory. Also, as the density of water is unity according to the C.G.S. system of units, derivations involving the mass of the raindrops become easier if this system is used. Thus, we will use the C.G.S. unit system in this paper. According to Maxwell [1], we know that when a water droplet grows in an undisturbed gaseous environment, steady state diffusion of water vapor takes place towards the droplet. Under such a condition, Laplace's equations are satisfied:

$$
\nabla^{2} \rho=0
$$

and

$$
\nabla^{2} T=0
$$

with $\rho$ and $T$ being the density and temperature respectively. The amount of water vapor diffusing per unit time to the surface of the spherical droplet can be calculated in the following manner.

Fick's first law of diffusion [13] says that the total mass of the molecules crossing per unit area per unit time is directly proportional to both the surface area and the gradient of concentration normal to it. So, if,

$$
\begin{array}{ll}
\mathrm{m}_{\mathrm{r}} & =\text { the mass of the droplet of radius ' } r \text { ', } \\
\mathrm{D} & =\text { the diffusion coefficient of water vapor in air, } \\
\mathrm{S} & =\text { the surface area of the droplet, } \\
\rho & =\text { ambient water vapor density, } \\
\rho_{\mathrm{r}} & =\text { the density of water vapor on the surface of the droplet, } \\
\rho_{\mathrm{r}}^{\prime} & =\text { the general water vapor density, } \\
\mathrm{r} & =\text { the radius of the droplet, } \\
\mathrm{r}^{\prime} & =\text { the radial coordinate, then we get, }
\end{array}
$$




$$
\frac{d m_{r}}{d t}=S D \frac{d \rho_{r}^{\prime}}{d r^{\prime}}=C_{1}
$$

where $C_{1}$ is a constant under stationary conditions. Now, the surface area of a spherical droplet is

$$
S=4 \pi r^{2}
$$

On integration with respect to the radial axis r', equation (3.3) yields,

$$
\rho_{r}^{\prime}=-\frac{C_{1}}{4 \pi r^{\prime} D}+C_{2}
$$

where $C_{2}$ is an arbitrary constant of integration.

Applying boundary conditions, at $r^{\prime}=\infty, \rho_{r}^{\prime}=\rho$, and at $r^{\prime}=r, \rho_{r}^{\prime}=\rho_{r}$ we obtain,

$$
C_{2}=\rho
$$

and

$$
\rho_{\mathrm{r}}^{\prime}=-\left(\rho-\rho_{\mathrm{r}}\right) \frac{\mathrm{r}}{\mathrm{r}^{\prime}}+\rho
$$

Equation (3.7) is of hyperbolic nature with respect to $r$ '. So,

$$
\frac{d p_{r}^{\prime}}{d r^{\prime}}=\frac{\left(p-p_{r}\right) r}{r^{\prime 2}}
$$

A combination of equations (3.3), (3.4) and (3.8) yields,

$$
\frac{d m_{r}}{d t}=4 \pi r D\left(\rho-\rho_{r}\right)
$$

Equation (3.9) may be also rewritten, relating the rate of mass-transfer with ambient vapor pressure (p) and the pressure at the surface of the droplet $\left(p_{r}\right)$, as

$$
\frac{d m_{t}}{d t}=4 \pi r k_{1} \Delta p
$$

with $\mathrm{k}_{1}$ being a constant and $\Delta \mathrm{p}=\mathrm{p}-\mathrm{p}_{\mathrm{r}}$.

Now, if $S_{r}$ be the mass of the dissolved gas, the rate of dissolution is given by

$$
\frac{d S_{r}}{d t}=4 \pi r^{2} A \mu
$$

with $\mu$ as the mass of gas striking per unit area per unit time and ' $A$ ', the fraction captured.

Again, the mass of the droplet is (the density of water being unity)

$$
\mathrm{m}_{\mathrm{T}}=\frac{4}{3} \pi \mathrm{r}^{3}
$$

The concentration of the dissolved gas, $\mathrm{C}_{r}$, is then,

$$
C_{T}=\frac{S_{T}}{m_{T}}
$$

So,

$$
\begin{aligned}
\frac{d C_{T}}{d t} & =\frac{1}{m_{\mathrm{T}}}\left[\frac{d S_{\mathrm{r}}}{d t}-\frac{S_{\mathrm{r}}}{m_{\mathrm{T}}} \frac{d \mathrm{~m}_{\mathrm{r}}}{\mathrm{dt}}\right] \\
& =\frac{3}{4 \pi \mathrm{r}^{3}}\left[4 \pi \mathrm{r}^{2} \mathrm{A \mu}-\mathrm{C}_{\mathrm{T}} \cdot 4 \pi \mathrm{r} \mathrm{k}_{1} \Delta \mathrm{p}\right] \\
& =\frac{3 \mathrm{A \mu}}{\mathrm{r}}-\frac{3 \mathrm{k}_{1} \Delta \mathrm{p} \mathrm{C}_{\mathrm{T}}}{\mathrm{r}^{2}}
\end{aligned}
$$


We now relate the fraction of gas molecules captured by the droplet to its capacity to retain it. As the concentration of dissolved gas approaches saturation, this capacity decreases. Thus,

$$
A=a\left(C_{s}-C_{t}\right)
$$

where $\mathrm{C}_{\mathrm{s}}$ is the solubility of the gas at the given temperature and pressure, and ' $\mathrm{a}$ ' is a constant. Again, equation (3.14) can be rewritten as

$$
\begin{aligned}
& \frac{d C_{I}}{d t}=\frac{3 a\left(C_{s}-C_{r}\right) \mu}{r}-\frac{3 k_{1} \Delta p C_{I}}{r^{2}} \\
& =\frac{3 a \mu C_{8}}{r}-\frac{3 C_{C}}{r}\left(a \mu+\frac{k_{1} \Delta p}{r}\right) \\
& \text { or, } \quad \frac{\mathrm{dC}_{\mathrm{r}}}{\mathrm{dt}}+\frac{3 \mathrm{C}_{\mathrm{r}}}{\mathrm{r}}\left(\mathrm{a} \mu+\frac{\mathrm{k}_{1} \Delta \mathrm{p}}{\mathrm{r}}\right)-\frac{3 \mathrm{a} \mu \mathrm{C}_{\mathrm{s}}}{\mathrm{r}}=0
\end{aligned}
$$

The above expression can be combined with equations (3.9) and (3.10) to give us

or,

$$
\begin{aligned}
& \frac{d C_{T}}{d t}+\frac{3 D\left(\rho-\rho_{r}\right) C_{T}}{r^{2}}-\frac{3 a \mu\left(C_{s}-C_{r}\right)}{r}=0 \\
& \frac{d C_{r}}{d t}+\left[\frac{3 D\left(\rho-\rho_{r}\right)}{r^{2}}+\frac{3 a \mu}{r}\right] C_{T}-\frac{3 a \mu}{r} C_{s}=0
\end{aligned}
$$

Now, let

$$
\left[\frac{3 \mathrm{D}\left(\rho-\rho_{\mathrm{r}}\right)}{\mathrm{r}^{2}}+\frac{3 \mathrm{a} \mu}{\mathrm{r}}\right]=\alpha
$$

and

$$
\frac{3 a \mu}{r} C_{s}=\beta \text {, }
$$

so, equation (3.18) becomes

or,

$$
\begin{aligned}
& \frac{d C_{T}}{d t}+\alpha C_{T}-\beta=0 \\
& \frac{d}{d t}\left(C_{r} e^{\alpha t}\right)=\beta e^{\alpha t}
\end{aligned}
$$

Near saturation, the densities and the radius are almost constant with time. At such times, $\alpha$ and $\beta$ are independent of $t$, as $r$ is constant. Then, on integration of equation (3.20), we get,

$$
C_{r}=\frac{\beta}{\alpha}\left(1-e^{-\alpha}\right)
$$

The above equation gives us the variation of $C_{r}$ with time $t$, i.e. the time rate of change of concentration of the dissolved gases. It can be expected that the first term on the right will have dominance over the second upto a certain time interval after which the second one involving the negative exponential of time becomes predominant. A graph resulting from our above derivation is found to be in good agreement with Rogers and Yau's [14] result obtained for the nucleation of liquid water in water vapor.

In the special case where $\Delta p \rightarrow 0$, according to equation (3.10), we find that $m_{r}$ becomes constant. Hence the radius of the droplet, $r$, becomes constant. So no further growth of the drop by condensation is possible. Accordingly, equation (3.16) becomes

$$
\frac{d C_{r}}{d t}=\frac{3 a\left(C_{s}-C_{r}\right) \mu}{r}
$$

which can also be written as

$$
-\frac{d\left(C_{s}-C_{r}\right)}{d t}=\frac{3 a\left(C_{s}-C_{r}\right) \mu}{r}
$$


Rearrangement, integration and application of boundary condition to equation (3.23) yields

$$
C_{T}=C_{s}\left[1-\exp \left(\frac{-3 a \mu t}{r}\right)\right]
$$

(3.24) shows the variation of $\mathrm{C}_{\mathrm{r}}$ with time if there is no growth of the droplet by condensation.

Let us now discuss about the other important factor, the variation of concentration of the pollutant gas with the growth and/or evaporation (size) of the droplet.

$$
\begin{aligned}
\frac{d C_{r}}{d r} & =\frac{d}{d r}\left(\frac{S_{r}}{m_{r}}\right) \\
& =\frac{3 a \mu\left(C_{s}-C_{r}\right)}{D\left(\rho-\rho_{r}\right)}-\frac{3 C_{r}}{r} \\
& =-3 C_{r}\left[\frac{a \mu}{D\left(\rho-\rho_{r}\right)}+\frac{1}{r}\right]+\frac{3 a \mu C_{s}}{D\left(\rho-\rho_{r}\right)} \\
\frac{d C_{T}}{d r} & +3 C_{r}\left[\frac{a \mu}{D\left(\rho-\rho_{r}\right)}+\frac{1}{r}\right]-\frac{3 a \mu C_{s}}{D\left(\rho-\rho_{r}\right)}=0
\end{aligned}
$$

or,

Therefore,

$$
\frac{d}{d r}\left(C_{r} \cdot r^{3} \exp \left[\frac{3 a \mu r}{D\left(\rho-\rho_{t}\right)}\right]\right)=\frac{3 a \mu C_{t}}{D\left(\rho-\rho_{t}\right)} \cdot r^{3} \exp \left[\frac{3 a \mu r}{D\left(\rho-\rho_{r}\right)}\right]
$$

The above equation has a solution of the following form

$$
\begin{aligned}
& C_{r} \cdot r^{3} \exp \left[\frac{3 a \mu r}{D\left(\rho-\rho_{r}\right)}\right] \\
& =\frac{3 a \mu C_{s}}{D\left(\rho-\rho_{r}\right)}\left[r^{3}-\frac{3 D\left(\rho-\rho_{r}\right)}{3 a \mu} r^{2}+\frac{6 D^{2}\left(\rho-\rho_{r}\right)^{2}}{9 a^{2} \mu^{2}} r-\frac{6 D^{3}\left(\rho-\rho_{r}\right)^{3}}{27 a^{3} \mu^{3}}\right] \frac{D\left(\rho-\rho_{r}\right)}{3 a \mu} \cdot \exp \left(\frac{3 a \mu r}{D\left(\rho-\rho_{r}\right)}\right)+\delta
\end{aligned}
$$

where $\delta$ is a constant of integration.

Equation (3.26) leads us to

$$
C_{r}=\frac{C_{8}}{r^{3}}\left[r^{3}-\frac{3 D\left(\rho-\rho_{r}\right)}{3 a \mu} r^{2}+\frac{6 D^{2}\left(\rho-\rho_{t}\right)^{2}}{9 a^{2} \mu^{2}} r-\frac{6 D^{3}\left(\rho-\rho_{t}\right)^{3}}{27 a^{3} \mu^{3}}\right]+\frac{\delta}{r^{3}} \cdot \exp \left(\frac{-3 a \mu_{r}}{D\left(\rho-\rho_{t}\right)}\right)
$$

From above, we get a relation between $\mathrm{C}_{\mathrm{S}}$ and $\mathrm{C}_{\mathrm{r}}$ as

$$
C_{I}=C_{s}\left[1-\frac{D\left(p-p_{r}\right)}{a \mu r}+\frac{2}{3} \cdot \frac{D^{2}\left(p-p_{r}\right)^{2}}{a^{2} \mu^{2} r^{2}}-\frac{2}{9} \cdot \frac{D^{3}\left(p-p_{t}\right)^{3}}{a^{3} \mu^{3} r^{3}}\right]+\frac{\delta}{r^{3}} \cdot \exp \left(\frac{-3 a \mu r}{D\left(p-p_{r}\right)}\right)
$$

Application of boundary conditions yields the constant $\delta$ given by

$$
\begin{aligned}
\delta & =e^{-\alpha t} \\
& =\exp \left[-\left\{\frac{3 \mathrm{D}\left(\rho-\rho_{\mathrm{r}}\right)}{\mathrm{r}^{2}}+\frac{3 \mathrm{a} \mu}{\mathrm{r}}\right)\right] \mathrm{t}
\end{aligned}
$$

Equation (3.27) gives us the relation of $C_{r}$ with time. The equation also shows how $C_{r}$ varies with the variation of the droplet radius.

Combining equation (3.15) and (3.27) we also find

$$
A=a C_{s}\left[\frac{D\left(\rho-\rho_{r}\right)}{a \mu r}-\frac{2}{3} \cdot \frac{D^{2}\left(\rho-\rho_{t}\right)^{2}}{a^{2} \mu^{2} r^{2}}+\frac{2}{9} \cdot \frac{D^{3}\left(\rho-\rho_{r}\right)^{3}}{a^{3} \mu^{3} r^{3}}\right]-\frac{\delta}{r^{3}} \cdot \exp \left(\frac{-3 a \mu_{r}}{D\left(\rho-\rho_{z}\right)}\right)
$$


We will proceed to evaluate the constant ' $a$ ' in equation (3.15) in the following section.

The equations (3.1) - (3.15) are valid even when the concentration of the gas is high enough that the concept of mass-transfer by diffusion is applicable. These equations are equally applicable to cloud droplets and raindrops as long as there is no collision between them.

Following Hales' [5] approach, we write a convective-diffusive mass-transfer equation as

$$
\begin{aligned}
A \mu & =a \mu\left(C_{s}-C_{r}\right) \\
& =a^{\prime}\left(C_{s}-C_{r}\right)
\end{aligned}
$$

with $\mathrm{a}^{\prime}=\mathbf{a \mu}$.

According to Chang [15] the mass flux of any gas to a droplet is given by

$$
\mathrm{F}=\mathrm{K}_{\mathrm{g}}\left(\mathrm{C}_{\mathrm{g}}-\mathrm{C}_{\mathrm{g}}^{*}\right)
$$

where $F=$ mass flux of the gas to the droplet,

$C_{B}^{*}=$ bulk gas phase concentration of the gas,

$\mathbf{C}_{\mathbf{g}}$ = concentration of the gas in equilibrium with the aqueous concentration of the dissolved gas at the surface of the droplet, and

$\mathrm{K}_{\mathbf{g}}=$ convective-diffusive mass-transfer coefficients.

Again, with reference to Bird et al [16], we can write $\mathrm{K}_{\mathrm{g}}$ as Frössling correlation [17] as

$$
\mathrm{K}_{\mathrm{g}}=\frac{2 \mathrm{D}_{\mathrm{g}}}{\mathrm{D}_{0}}\left(1+0.3 \operatorname{Re}_{2}^{\frac{1}{2}} \mathrm{Sc}_{3}^{1}\right)
$$

where

$\mathrm{D}_{\mathrm{g}}=$ diffusivity of the gas in air,

$\mathrm{D}_{\mathrm{o}}=$ diameter of a sphere of the same volume as a falling raindrop,

$\mathrm{Re}=$ Reynold's number $=\frac{2 \mathrm{rV}}{\mathrm{V}}$, and

$\mathrm{Sc}=$ Schmidt's number $=\frac{\underset{v}{V}}{\mathrm{DA}_{\mathbf{y}}}$,

$\mathrm{DA}_{\mathrm{y}}$ being the diffusion coefficient of the gas, $\mathrm{V}$ the terminal velocity of the droplet and $\mathrm{v}$ the kinematic viscosity of air.

An alternate form of equation (3.32) is

$$
K_{g}=\frac{D_{g}}{D_{0}} S_{h}
$$

where $S_{h}$ is the Sherwood's number [18] $=2+0.6 \operatorname{Re}_{2}^{\frac{1}{2}} \operatorname{Sc}_{3}^{\frac{1}{3}}$

It should be noted here that equations (3.30) and (3.31) are of the same nature with the only difference that while the former one is applicable to processes involving a discontinuous medium, the latter one is applicable to those involving a continuous medium.

\section{CORRESPONDENCE BETWEEN EQUATIONS (3.30) AND (3.31).}

We will answer the following question: how is the equation applicable to the discontinuous medium related to the one applicable to the continuous medium? Henry's law [13] states that the mole fraction of a dissolved gas (in a liquid) is proportional to the mole fraction of the gas (i.e. the partial pressure of the gas) in gaseous mixtures in contact with it at equilibrium. Since mole fraction of a gas is proportional to concentration, we may write $X_{s}$ is proportional to $C_{g}$ where $X_{s}$ is the mole fraction of the dissolved gas saturated in the ambient condition of temperature and partial pressure. Hence,

$$
\mathrm{X}_{\mathrm{s}}=\mathrm{HC}_{\mathrm{g}}
$$

where $\mathrm{H}$ is called Henry's constant.

Under normal conditions, the pollutant gas in a saturated solution of rain or cloud should be dilute.

Hence, by definition, 


$$
x_{s}=\frac{n}{n+N} \approx \frac{n}{N}
$$

where $\mathrm{n}$ is the number of moles of solute (dissolved gas) in $\mathbf{N}$ (the number of moles of solvent). But,

$$
\mathbf{n}=\frac{\mathbf{S}_{\mathbf{r}}}{\mathbf{M}}
$$

where $\mathrm{M}$ is the molecular weight of the dissolved gas.

Again, $N=\frac{M_{T}}{18}$ (molecular weight of water being 18 ). Therefore,

$$
X_{r}=\frac{n}{N}=\frac{\frac{S_{r}}{M}}{\frac{M_{r}}{18}}=\frac{18 C_{r}}{M}
$$

where $\mathrm{X}_{\mathrm{r}}$ is the mole fraction of dissolved gas in droplet of radius ' $r$ '. Similarly, we get

$$
X_{s}=\frac{18 C_{s}}{M}
$$

Thus, from equations (4.1), (4.4) and (4.5), we get

$$
\begin{aligned}
& \mathrm{HC}_{\mathrm{g}}=\frac{18 \mathrm{C}_{\mathrm{s}}}{\mathrm{M}} \text {, and } \mathrm{HC}_{\mathrm{g}}^{*}=\frac{18 \mathrm{C}_{\mathrm{r}}}{\mathrm{M}} \\
& \text { or, } \mathrm{C}_{\mathrm{s}}=\frac{\mathrm{MH}}{18} \mathrm{C}_{\mathrm{g}} \text {, and } \mathrm{C}_{\mathrm{r}}=\frac{\mathrm{MH}}{18} \mathrm{C}_{\mathrm{g}}^{*}
\end{aligned}
$$

Mass flux of a gas given by equation (3.31) is $\mathrm{F}$, which should correspond to the rate of capture of mass of gas by unit area of the droplet as given by equation (3.11). Thus,

$$
F=\frac{1}{4 \pi r^{2}} \frac{d S_{r}}{d t}=A \mu=a^{\prime}\left(C_{s}-C_{r}\right)
$$

Thus,

[using equation (3.30)]

$$
F=a^{\prime}\left(C_{s}-C_{T}\right)=\frac{a^{\prime} M H}{18}\left(C_{g}-C_{g}^{*}\right)
$$

Comparing equations (3.31) and (4.7) we conclude that

$$
\frac{\mathrm{a}^{\prime} \mathrm{MH}}{18}=\mathrm{K}_{\mathbf{B}}
$$

Rearrangement gives,

$$
\begin{aligned}
& a^{\prime}=\frac{18 K_{g}}{M H} \\
& \text { or, } \\
& a=\frac{18 \mathrm{~K}_{\mathbf{g}}}{\mu \mathrm{MH}}
\end{aligned}
$$

\section{CONCLUSIONS.}

Equation (4.8) gives us an expression for ' $a$ ', the unknown constant in equation (3.15), which is obtained by treating the pollutant gas as a continuous medium. One can easily find out the value of $\mu$ (experimentally), and all other quantities on the right hand side of the equation are known. For example, equation (3.33) gives $K_{g}, M$ (the molecular weight of the dissolved gas) and $H$ (the Henry's constant ). So, in this way, we can get a good correlation between the diffusion approach and kinetic theory approach to the problem of gas-scavenging by rain. The value of ' $A$ ' may also be calculated with the help of the value of ' $a$ ' obtained from equation (3.15). 


\section{ACWNOWLEDGEMENTS.}

The authors acknowledge the helpful suggestions of Professors T.C. Roy and D.K. Bhattacharjee (of Department of Physics, Jadavpur University, Calcutta, India) and Professors John. E. Bauman Jr. and Patricia L. Moore Plummer (of Departments of Chemistry and Physics, University of Missouri-Columbia, Columbia, Missouri, U.S.A.) at different stages of this work. The authors would also like to thank Mrs. Durba Chattopadhyay and Ms. Brenda Frazier for their help with typing and proof-reading the manuscript.

Communiactions regarding this paper should be addressed to SC. AKM has recently retired as the additional director general of the India Meteorological Services, and a professor of Physics and Meteorology of Indian Institute of Technology, Kharagpur.

\section{REFERENCES}

1. MAXWELL, J. C., The Scientific Papers of James Clerk Maxwell,2, Dover, New York, (1890), 636640.

2. MASON, B. J., The Physics of Clouds, 2nd edition, Oxford U. Press, (1971), 84.

3. NEIBERGER, M., Proc. Fifth Berkeley Symp. Math. Stat. and Prob. ,University of California, Berkeley, (1967), 1-27.

4. JIUSTO, J. E., PILLIE, and R. J., KOCMOND, R. C., J App. Meteor.7, (1968), 860-869.

5. HALES, J.M., Atmos. Environ., 6, (1972), 635-659.

6. ENGELMANN, R.J., Scavenging meteorology and atomic energy, ed. D.H. Slade, USA EC 6860097, (1968), 208.

7. FUQUAY, J.J., Precipitation Scavenging, ed.R.J. Engelmann and W.G.N.Slinn (1970), 1.

8. JUNGE, C. E., Air Chemistry and Radioactivity, Academic, New York, (1963), 291.

9. POSTMA, A., Precipitation Scavenging, ed. R.J. Engelmann and W.G.N. Slinn, (1970),.267.

10. HALES, J. M., THORP and J. M., WOLF, M. A. Final Report to EPA. Number CPA 22-69-150, U.S.A. (1971).

11. FUKUTA, N., WALTER, L. A., J.Atmos. Sc. 27, (1970), 1160-1172.

12. MUKHERJEE, A. K., Communications a'la VIII eme Conference Internationale Sur la Physique des nuages , 1, (1980), 169-172; MUKHERJEE A. K. and KRISHNA NAND, Indian J. Met. Geophy.(Mausam), 32, (1981) 231-236.

13. See for example, P. W. Atkins, Physical Chemistry, Freeman, San Francisco (1982).

14. ROGERS,R.R. and YAU,M.K., A short course in cloud physics. Pergamon, (1989), 84.

15. CHANG, T. Y., Atmos. Environ, 18, (1984), 191-197.

16. BIRD, R.B., STEWARD, W. E., and LIGHTFOOT, E. N., Transport Phenomena, Wiley, New York, (1964), 409.

17. FRÖSSLING, N., Gerlands Beitr. Geophys., 52, (1938), 170.

18. SHERWOOD, T. K., PIGFORD, R. L., Absorption and Extraction, McGrew-Hill, New York, (1952), 317. 


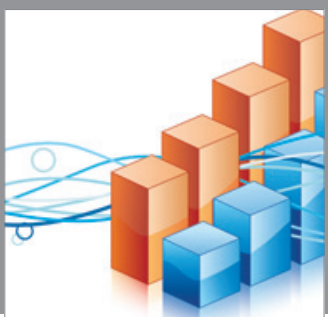

Advances in

Operations Research

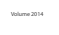

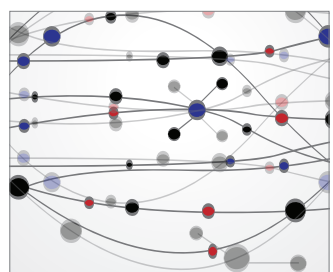

\section{The Scientific} World Journal
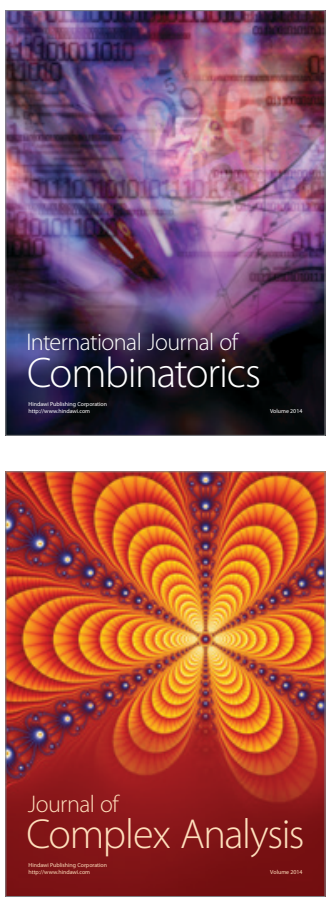

International Journal of

Mathematics and

Mathematical

Sciences
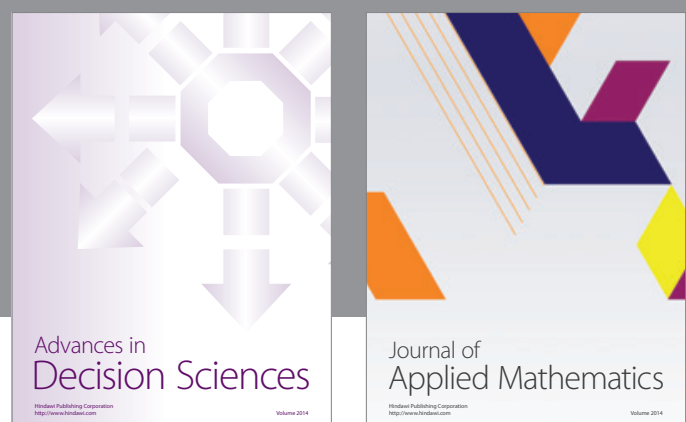

Journal of

Applied Mathematics
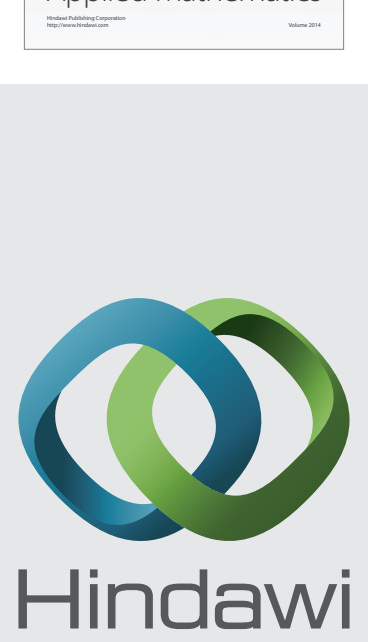

Submit your manuscripts at http://www.hindawi.com
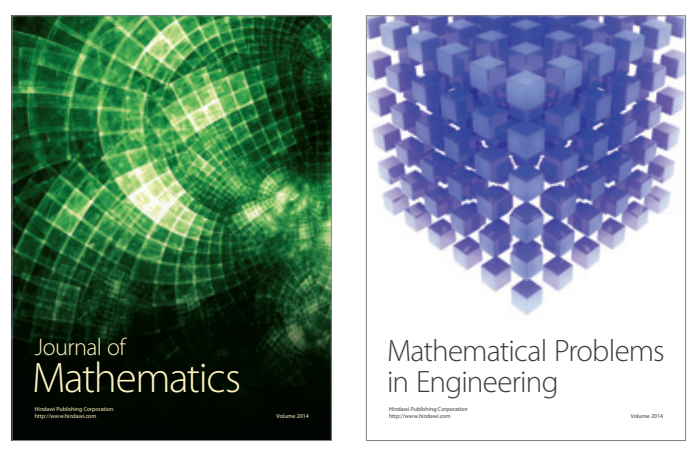

Mathematical Problems in Engineering
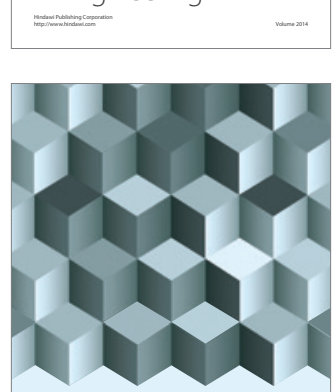

Journal of

Function Spaces
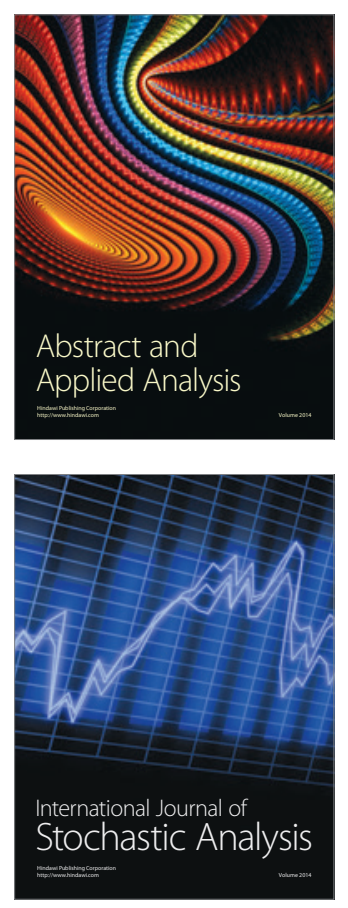

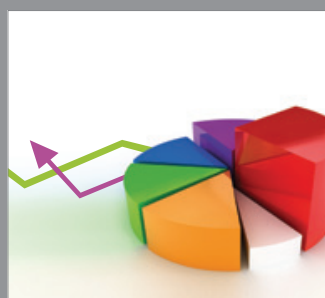

ournal of

Probability and Statistics

Promensencen
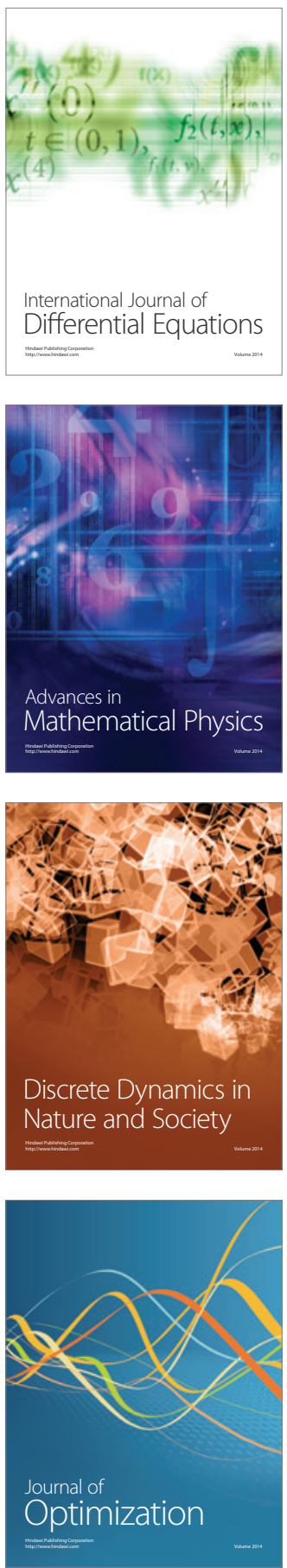